\title{
Association of Adverse Drug Events with Broad-spectrum Antibiotic Use in Hospitalized Patients: A Single-center Study
}

\author{
Hideharu Hagiya ${ }^{1}$, Ryohei Kokado ${ }^{1,2}$, Akiko Ueda ${ }^{3}$, Hideo Okuno ${ }^{1}$, Daiichi Morii ${ }^{1}$, \\ Shigeto Hamaguchi ${ }^{1}$, Norihisa Yamamoto ${ }^{1}$, Hisao Yoshida ${ }^{1}$ and Kazunori Tomono ${ }^{1}$
}

\begin{abstract}
:
Objective The importance of antimicrobial stewardship is increasingly highlighted in this age of antimicrobial resistance. A better comprehension of adverse drug events (ADEs) can promote the appropriate use of antibiotics. We aimed to quantify the incidence of ADEs associated with broad-spectrum systemic antibiotics in a hospital setting.

Methods We conducted a six-month prospective, observational study at Osaka University Hospital to describe the incidence of ADEs in patients hospitalized in general wards undergoing treatment with broadspectrum antibiotics [carbapenems, piperacillin/tazobactam (PIPC/TAZ), and anti-methicillin-resistant Staphylococcus aureus agents]. The occurrence of $\mathrm{ADE}$ was defined as any cardiac, gastrointestinal, hepatobiliary, renal, neurologic, hematologic, dermatologic, or musculoskeletal manifestation after 48 hours or more of systemic antibiotic therapy.

Results The 3 most frequently prescribed antibiotics were PIPC/TAZ (242 cases), meropenem (181 cases), and vancomycin (92 cases). Of 689 patients, 118 (17.1\%) experienced ADEs, including gastrointestinal $(6.4 \%)$, hepatobiliary $(4.2 \%)$, dermatologic $(2.5 \%)$, and renal $(2.3 \%)$ manifestations. Patients treated with PIPC/TAZ, meropenem, doripenem, vancomycin, daptomycin, and teicoplanin developed ADEs at rates of $20.7 \%, 16.0 \%, 15.4 \%, 19.6 \%, 11.8 \%$, and $10.9 \%$, respectively.

Conclusion Our study provides a quantitative value for the incidence of ADEs associated with broadspectrum antibiotics in clinical practice. To optimize patient safety, clinicians need to be aware of the risks associated with antibiotic administration.
\end{abstract}

Key words: choosing wisely, adverse drug event, antibiotic stewardship, antimicrobial resistance

(Intern Med 58: 2621-2625, 2019)

(DOI: 10.2169/internalmedicine.2603-18)

\section{Introduction}

The development of antibiotic therapy represents a significant medical achievement that is integral to modern medicine. At present, about half of hospitalized patients undergo at least 1 antibiotic treatment during their admission (1), which is estimated to be unnecessary in $20-30 \%$ of these patients (2). The reasons for overprescribing antibiotics include inappropriate medical indications (noninfectious diseases), improper targets (colonization or contaminations), and treatment durations that are longer than generally recommended. In addition to concerns over the emergence of antimicrobialresistant organisms (3), the excessive use of antibiotics can provoke adverse consequences.

Adverse drug events (ADEs) are clinically significant issues relating to any antibiotic prescription. The avoidance of ADEs is critical in hospitalized patients who are generally ill with various clinical complications. To minimize antibiotic-associated ADEs, an estimation of their incidence

${ }^{1}$ Division of Infection Control and Prevention, Osaka University Hospital, Japan, ${ }^{2}$ Department of Pharmacy, Osaka University Hospital, Japan and ${ }^{3}$ Laboratory for Clinical Investigation, Osaka University Hospital, Japan

Received: December 29, 2018; Accepted: March 1, 2019; Advance Publication by J-STAGE: May 22, 2019

Correspondence to Dr. Hideharu Hagiya, highgear@hp-infect.med.osaka-u.ac.jp 
Table 1. Criteria for Antibiotic-associated Adverse Drug Events.

\begin{tabular}{|c|c|}
\hline Categories & Definition \\
\hline Gastrointestinal & $\begin{array}{l}\text { Diarrhea; }>3 \text { times of loose stools per day associated with antibiotic administration and documented as "diarrhea" in the } \\
\text { medical record. Nausea and vomiting; documented as antibiotic-associated "nausea and/or vomiting" in the absence of an } \\
\text { alternate explanation }\end{array}$ \\
\hline Hematologic & $\begin{array}{l}\text { Anemia (hemoglobin level }<10 \mathrm{~g} / \mathrm{dL} \text { ), leukopenia (white blood cell count }<4,500 \mathrm{cells} / \mu \mathrm{L} \text { ), or thrombocytopenia (platelet } \\
\text { count }<150 \times 10^{3} / \mu \mathrm{L} \text { ) with levels below patient's baseline and in the absence of bleeding or myelosuppressive therapies }\end{array}$ \\
\hline Hepatobiliary & $\begin{array}{l}\text { Cholestasis (total bilirubin level }>3 \mathrm{mg} / \mathrm{dL} \text { ) or transaminitis (aspartate transaminase or alanine transaminase level }>3 \\
\text { times patient's baseline) in the absence of existing hepatobiliary disease or recent biliary instrumentation }\end{array}$ \\
\hline Renal & $\begin{array}{l}\text { Increase in serum creatinine level }>1.5 \text { times patient's baseline in the absence of precipitating factors for acute kidney } \\
\text { injury such as sepsis or the receipt of intravenous contrast or other nephrotoxic agents }\end{array}$ \\
\hline Neurologic & $\begin{array}{l}\text { Altered mental status, peripheral neuropathy, or seizures in the absence of preexisting neurologic conditions, substance- } \\
\text { related toxic effects, or infectious syndromes }\end{array}$ \\
\hline Dermatologic & Rash, temporally associated with antibiotic administration with resolution on antibiotic discontinuation \\
\hline Cardiac & QTc $>440 \mathrm{~ms}$ in males or $>460 \mathrm{~ms}$ in females in the absence of preexisting arrhythmias, based on $\geq 2$ electrocardiograms \\
\hline Anaphylaxis & $\begin{array}{l}\text { Acute onset of respiratory compromise, hypotension, or end-organ dysfunction within minutes after initiation of } \\
\text { antibiotic administration, in the absence of an alternative explanation }\end{array}$ \\
\hline Myositis & Increase in creatine phosphokinase level $>5$ times patient's baseline, in the absence of existing myopathy or statin use \\
\hline $\begin{array}{l}\text { Clostridium difficile } \\
\text { infection }\end{array}$ & $\begin{array}{l}\text { Clinical signs and symptoms consistent with } C \text {. difficile infection in the setting of a positive testing result for } C \text {. difficile } \\
\text { toxins }\end{array}$ \\
\hline
\end{tabular}

Defined by referring to the previous literature with minor changes [2]

is essential, but studies regarding the prevalence of antibiotic-associated ADEs in actual clinical practice have been insufficient. Previous studies were based on administrative databases alone and limited to a single infectious syndrome (4) or antibiotic class $(5,6)$. A comprehensive analysis of the ADE incidence among hospitalized patients was performed only recently in a study that was limited to a single facility (2).

In the present study, we aimed to evaluate the incidence of antibiotic-associated ADEs using actual clinical data from our hospital, focusing on patients receiving broad-spectrum antibiotics.

\section{Materials and Methods}

This observational study was conducted at Osaka University Hospital, a 1,086-bed tertiary care facility in Japan. The need for informed consent was waived since the data were anonymized for antimicrobial stewardship activities.

The clinical data from patients administered broadspectrum antibiotics between January and June of 2018 (six months) were collected from digital records. We defined broad-spectrum antibiotics as carbapenems (meropenem, imipenem, doripenem, biapenem), piperacillin/tazobactam (PIPC/TAZ), and anti-methicilln-resistant Staphylococcus aureus (MRSA) drugs (vancomycin, teicoplanin, daptomycin, linezolid, and arbekacin). The use of topical or inhaled antibiotics was excluded. A daily case conference was held on the validity of using broad-spectrum antibiotics at the Division of Infection Control and Prevention. An infectious disease physician and a pharmacist adjudicated all potential associations between data abnormalities and the antibiotics in the context of their medical history and clinical course.

Criteria for the definition of antibiotic-associated ADEs (cardiac, gastrointestinal, hepatobiliary, renal, neurologic, he- matologic, dermatologic, anaphylactic, musculoskeletal manifestations, and Clostridium difficile infection) were defined based on the previous literature (Table 1) (2). Patient data regarding these events were observed until cessation of the antibiotic treatment. To avoid overestimating the incidence of ADEs in patients who were administered multiple antibiotics, each ADE was categorized under a single antibiotic based on its likelihood of causing the specific ADE, as has been done in the recent publication (2). For example, thrombocytopenia in a patient receiving a combination of linezolid and meropenem was regarded as a linezolidassociated ADE. For the analysis, we included only cases treated in the general ward and excluded those treated in the intensive-care units in order to avoid the possibility of multiple factors leading to ADEs. Aside from an anaphylactic event, all manifestations that developed in patients undergoing $\geq 48$ hours of antibiotic treatment were included.

\section{Results}

During the study period, totally, antibiotics were administered to the patients as follows: PIPC/TAZ to 355, meropenem to 318 , doripenem to 58 , imipenem to 2 , vancomycin to 215 , teicoplanin to 61 , daptomycin to 63 , linezolid to 29 , and arbekacin to 2. Of them, $689(6.7 \%)$ out of 10,214 patients admitted to general ward of the hospital received broad-spectrum antibiotics (Table 2). The median age of the population was 61 years [interquartile range (IQR), 40-72 years], and the men-to-women ratio was 1.6 (421 to 268).

Of these 689 patients, $118(17.1 \%)$ experienced some type of ADE. Common manifestations included gastrointestinal $(6.4 \%)$, hepatobiliary $(4.2 \%)$, dermatologic $(2.5 \%)$, and renal $(2.3 \%)$ involvements. The most frequently prescribed antibiotics were PIPC/TAZ (242 cases), followed by meropenem (181 cases) and vancomycin (92 cases). Of these, 


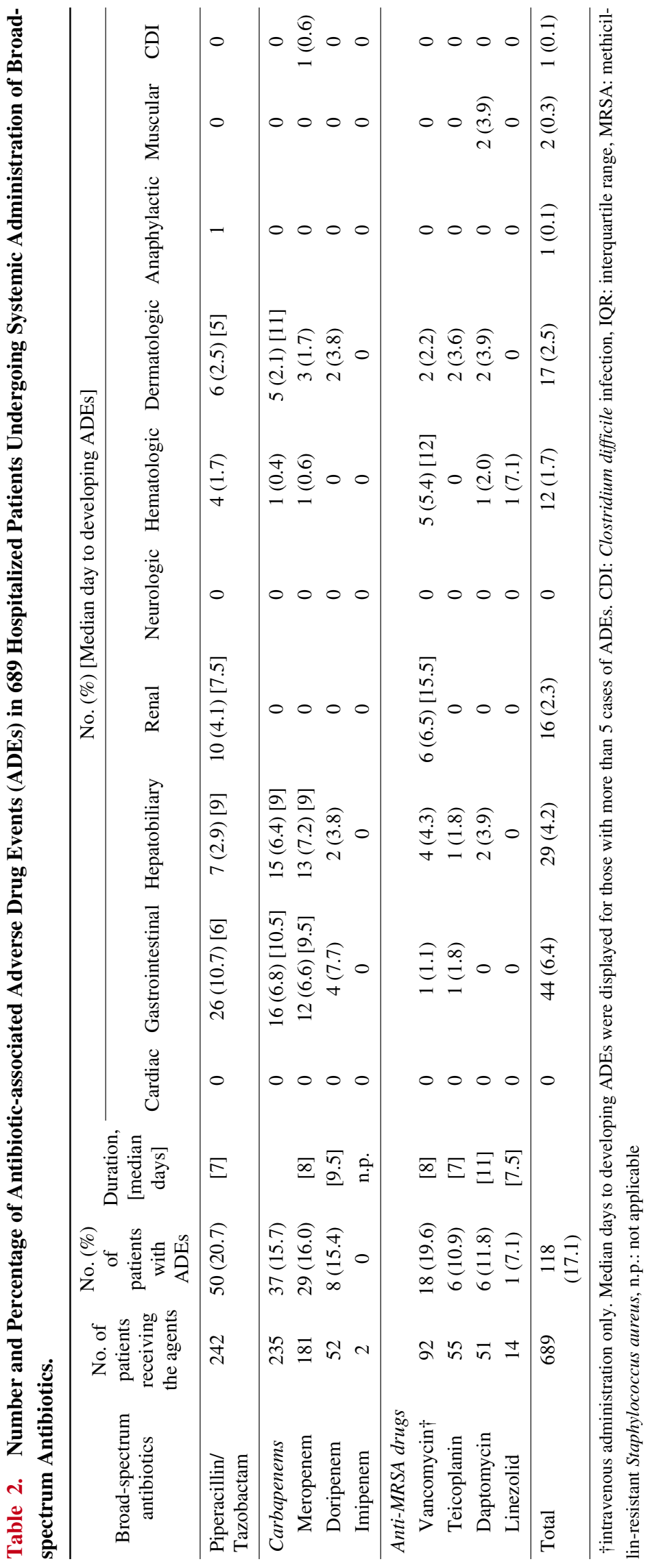

ADEs occurred most frequently with PIPC/TAZ, manifesting tologic (2.5\%) abnormalities. There was an anaphylactic in 50 of the 242 patients $(20.7 \%)$ as gastrointestinal case associated with PIPC/TAZ administration. Patients $(10.7 \%)$, renal $(4.1 \%)$, hepatobiliary $(2.9 \%)$, and derma- given meropenem developed ADEs in 29 of 181 instances 
$(16.0 \%)$ classified as hepatobiliary $(7.2 \%)$, gastrointestinal $(6.6 \%)$, and dermatologic $(1.7 \%)$ disorders. Patients given doripenem also developed ADEs with a similar pattern. Regarding anti-MRSA agents, vancomycin-induced ADEs occurred most frequently (19.6\%), followed by daptomycin $(11.8 \%)$ and teicoplanin $(10.9 \%)$. The 3 most common ADEs associated with vancomycin treatment were renal $(6.5 \%)$, hematologic $(5.4 \%)$, and hepatobiliary $(4.3 \%)$ abnormalities. The hematologic disorder in all five patients treated with vancomycin was leukocytopenia. Teicoplaninassociated ADEs were less common than vancomycinassociated ADEs (10.9\% vs. 19.6\%). Daptomycin caused myositis in two patients and linezolid-induced thrombocytopenia in one patient. There were no patients with cardiac or neurological manifestations. One patient administered meropenem developed Clostridium difficile infection.

\section{Discussion}

This study was performed under the umbrella of inhospital infection control activities in order to underscore the importance of antimicrobial stewardship. Our study revealed that $17.1 \%$ of patients hospitalized in the general ward (118 of 689 cases) developed ADEs that were associated with the administration of broad-spectrum antibiotics. This result mirrors the finding of a recent report (2), which showed that $20 \%$ of admitted patients treated with antibiotic therapy developed an antibiotic-associated ADE. Our study also highlights the high incidence of antibiotic-associated ADEs, suggesting a clinical need to reduce unnecessary treatment with broad-spectrum antibiotics.

Each of the preceding studies addressing antibioticassociated ADEs had several limitations. According to a previous study based on an administrative database, only $0.45 \%$ to $0.6 \%$ of patients hospitalized with pneumonia developed antibiotic-associated ADEs (4). However, the results were derived from registration data and therefore had undeniable under-reporting of existing ADEs. Furthermore, the authors focused only on patients with respiratory infections, so their results cannot be fully generalized. Another study based on a medical record review demonstrated that $27 \%$ of fluoroquinolone use was linked to ADEs, such as gastrointestinal disorders $(14 \%)$, colonization of multi-drug resistant organisms (8\%) and $C$. difficile infections (4\%) (6). The study period was a mere six weeks, and the authors focused only on fluoroquinolone use, limiting the versatility of the data.

In general, hospitalized patients are more vulnerable to ADEs than outpatients for several reasons. First, inpatients usually undergo intravenous antibiotic therapy at higher doses than oral regimens, possibly leading to a high incidence of ADEs (7). Second, the combined use of multiple medications is more frequent in inpatients than in outpatients, potentially resulting in interactive drug reactions (8). Third, inpatients are generally older with multiple underlying diseases and decreased drug excretion (9). However, we should be mindful of antibiotic-associated ADEs in the out- patient cohort as well. According to a previous study assessing the frequency of drug-related adverse events in emergency department visits, antibiotic prescriptions accounted for $19.3 \%$ of cases (10). Thus, it is important to recognize that antibiotics introduce a potential risk of ADEs with high frequency in any medical situation.

Our study has the advantage of being relevant to the scientific community. In contrast to previous studies, we performed the present study in a prospective manner by focusing on the occurrence of broad-spectrum antibioticassociated ADEs as part of the Antimicrobial Stewardship Program. However, several limitations should also be mentioned. First, the single-center nature of the miscellaneous patient population should be considered when generalizing the results to other medical settings. Second, reviewing medical charts may have led to an underestimation of ADEs, although we believe this approach is more appropriate and reliable than using an administrative database, which includes the possibility of miscoding. Conversely, the number of antibiotic-associated ADEs may have been overestimated because cases of ADEs caused by non-antibiotic drugs were also considered. Third, the case numbers were relatively few, and a more accurate estimate would be obtained from a larger-scale study. Fourth, other clinically common ADEs, including drug fever and eosinophilia, were not included in the study criteria. Finally, data on the treatment duration were lacking.

Collectively, the overall incidence rate of broad-spectrum antibiotic-associated ADEs was $17.1 \%$ for patients hospitalized in the general ward. Due to their varied manifestations, antibiotic-associated ADEs are often under-recognized by clinicians. However, the occurrence of antibiotic-associated ADEs cannot be ignored for inpatients, as they pose a high clinical burden for this patient group. To optimize patient safety, infection control practitioners and clinicians should pay close attention to ADEs as part of their antimicrobial stewardship activities.

The authors state that they have no Conflict of Interest (COI).

\section{References}

1. Pretorius RW, Gataric G, Swedlund SK, Miller JR. Reducing the risk of adverse drug events in older adults. Am Fam Physician 87: 331-336, 2013.

2. Tamma PD, Avdic E, Li DX, Dzintars K, Cosgrove SE. Association of adverse events with antibiotic use in hospitalized patients. JAMA Intern Med 177: 1308-1315, 2017.

3. Bell BG, Schellevis F, Stobberingh E, Goossens H, Pringle M. A systematic review and meta-analysis of the effects of antibiotic consumption on antibiotic resistance. BMC Infect Dis 14: 13, 2014.

4. Lin RY, Nuruzzaman F, Shah SN. Incidence and impact of adverse effects to antibiotics in hospitalized adults with pneumonia. J Hosp Med 4: E7-E15, 2009.

5. Macy E, Contreras R. Adverse reactions associated with oral and parenteral use of cephalosporins: a retrospective population-based analysis. J Allergy Clin Immunol 135: 745-752, 2015. 
6. Werner NL, Hecker MT, Sethi AK, Donskey CJ. Unnecessary use of fluoroquinolone antibiotics in hospitalized patients. BMC Infect Dis 11: 187, 2011.

7. File TM Jr, Segreti J, Dunbar L, et al. A multicenter, randomized study comparing the efficacy and safety of intravenous and/or oral levofloxacin versus ceftriaxone and/or cefuroxime axetil in treatment of adults with community-acquired pneumonia. Antimicrob Agents Chemother 41: 1965-1972, 1997.

8. Hammond DA, Smith MN, Li C, Hayes SM, Lusardi K, Bookstaver PB. Systematic review and meta-analysis of acute kidney injury associated with concomitant vancomycin and piperacillin/tazobactam. Clin Infect Dis 64: 666-674, 2017.
9. Martin RM, Biswas PN, Freemantle SN, Pearce GL, Mann RD. Age and sex distribution of suspected adverse drug reactions to newly marketed drugs in general practice in England: analysis of 48 cohort studies. Br J Clin Pharmacol 46: 505-511, 1998.

10. Shehab N, Patel PR, Srinivasan A, Budnitz DS. Emergency department visits for antibiotic-associated adverse events. Clin Infect Dis 47: 735-743, 2008.

The Internal Medicine is an Open Access journal distributed under the Creative Commons Attribution-NonCommercial-NoDerivatives 4.0 International License. To view the details of this license, please visit (https://creativecommons.org/licenses/ by-nc-nd/4.0/).

(C) 2019 The Japanese Society of Internal Medicine Intern Med 58: 2621-2625, 2019 